\title{
Cardiac surgery in a patient with essential thrombocythemia: a case report
}

\author{
B.E. Schölzel, H. Endeman, W. Dewilde, A. Yilmaz, O. de Weerdt, J.M. ten Berg
}

Patients with essential thrombocythemia (ET) are at increased risk of developing arterial thrombosis. We report a case of a 36-year-man with unstable angina in the presence of occlusion of two coronary arteries with insufficient collateral perfusion. We also found essential thrombocythemia in this patient. The patient underwent coronary artery bypass grafting $(\mathrm{CABG})$. Ten days before surgery, the aspirin was replaced by a prophylactic dose of low-molecular-weight heparin. Postoperative followup was complicated by pulmonary embolisms and a cardiac tamponade. We conclude that ET is a risk factor for coronary heart disease that should be treated with aspirin. If a patient needs CABG, aspirin should be continued because of the high risk of thromboembolic events in the high-risk ET patients. (Neth Heart J 2010;18:378-80.)

Keywords: Coronary Artery Bypass Grafting; Thrombocythemia, Essential; Aspirin;

Case Reports

$\mathbf{T}$ hrombotic complications and bleeding are major causes of illness and death in patients with essential thrombocythemia, particularly older patients and patients with cardiovascular risk factors. ${ }^{1-4}$ This is illustrated by the case we present of coronary bypass artery grafting $(\mathrm{CABG})$ surgery in a patient with essential thrombocythemia.

\section{B.E. Schölzel \\ H. Endeman \\ W. Dewilde \\ A. Yilmaz \\ O. de Weerdt \\ J.M. ten Berg}

Correspondence to: B.E. Schölzel

Department of Cardiology, St. Antonius Hospital, PO Box 2500, 3430 EM Nieuwegein, the Netherlands

E-mail: bscholzel@hotmail.com

\section{Case report}

A 36-year-old man visited our clinic with progressive chest pain. Risk factors for coronary heart disease (CHD) were overweight (BMI 29.1), a history of smoking and hypertension. The family history was negative for $\mathrm{CHD}$.

The electrocardiogram (ECG) at presentation was normal, but ECGs during exercise showed slight ST depression in leads V4 to V6, suggestive of coronary insufficiency. His triglycerides were elevated $(3.93 \mathrm{mmol} / \mathrm{l})$ and his HDL cholesterol was low $(0.77 \mathrm{mmol} / \mathrm{l})$. Therefore, the patient was treated with aspirin, a $\beta$-blocker and a statin. Despite this medication the patient remained symptomatic and myocardial scintigraphy was performed, showing large areas of ischaemia in the anterior, apical and inferior wall. Subsequent coronary angiography (CAG, figure 1 ) showed an occluded ramus descendens anterior and right coronary artery, without signs of vessel irregularities in the ramus circumflexus. There was a welldeveloped collateral circulation. The patient was accepted for CABG.

A full blood count performed before CAG showed a thrombocythemia (1438 x $10^{9} / 1$ platelets), without any evidence of a reactive cause. A bone marrow aspiration was done demonstrating many megakaryocytes (figure 2). The diagnosis of essential thrombocythemia (ET) was made and the patient was started on hydroxyurea on top of aspirin.

Ten days before CABG, the aspirin was replaced by a prophylatic dose of low-molecular-weight heparin. The CABG was severely complicated by clot formation in the coronary arteries. The clots were removed immediately by the surgeon. Immediately after the operation, oral anticoagulation and lowdose aspirin (100 mg daily) were started. Peroperatively the platelet count was above $1000 \times 10^{9} / 1$. The postoperative course was complicated by first a tamponade and later by a pulmonary embolus. After the pulmonary embolus, the dose of aspirin was 


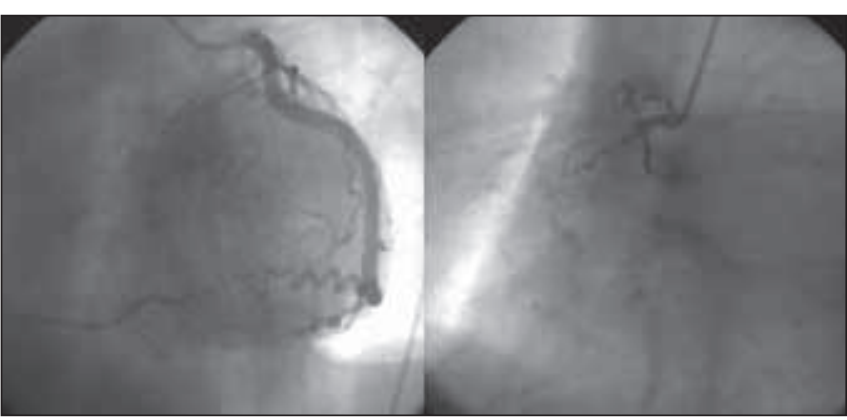

Figure 1. Occlusion of the ramus descendens anterior and right coronary artery, without signs of vessel irregularities in the ramus circumflexus.

increased to $300 \mathrm{mg}$ daily. Eventually the patient was sent home in a good clinical condition.

\section{Discussion}

ET is a rare disease $(2.5 / 100,000$ persons/year $)$, which is characterised by a high platelet count $\left(>600 \times 10^{9} / 1\right),{ }^{2}$ megakaryocytic hyperplasia in the bone marrow, and the absence of a reactive cause or another myeloproliferative syndrome. ${ }^{1}$ About 9 to $22 \%$ of the patients with ET present with a vascular event (venous and/or arterial event). The cardiovascular complication rate in patients with ET is between 4 and $21 \%{ }^{2}$ The exact mechanism responsible for this increased risk is elusive. However, a dysfunction of the thrombocytes is more likely to be the cause than just the increased number of platelets, because several reports have shown not only an elevated risk of thrombosis, but also an elevated risk of bleeding in patients with ET. ${ }^{3}$

This patient suffered from CHD due to a number of risk factors: a history of smoking, obesity, hypertension and ET. It is difficult to say which of these factors contributed most to his CHD, but with the finding of normal vessel walls in combination of two occluded coronary arteries during CAG, one could speculate that thromboembolism due to ET is responsible for his CHD. Although this may not reach much further than the level of speculation, it is quite reasonable to assign the peroperative thromboembolic events (easy coagulation during operation, tamponade and pulmonary embolus) to the ET.

Postoperatively the patient was treated with oral anticoagulation, but nevertheless he developed thrombosis and embolus. Aspirin was restarted, first in a low dose, but based on a minimal effect on the platelet function assay, later in high doses. Afterwards no new thromboembolic episodes occurred. In retrospect it would have been better to continue the aspirin during the cardiac surgery.

Previous reports have shown higher morbidity in patients with ET due to arterial ischaemic compli-

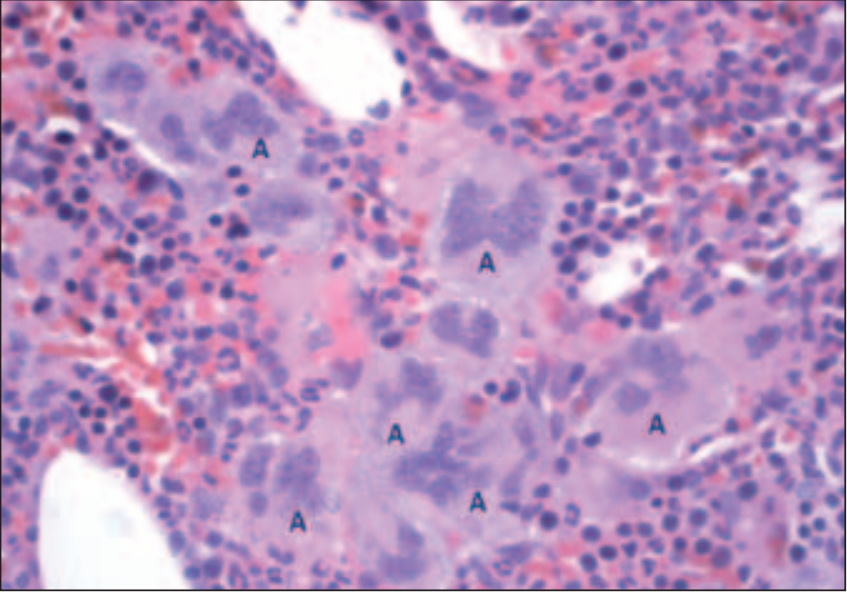

Figure 2. Histology of trephine bone marrow biopsy (H\&̈E stained) showing large clusters of megakaryocytes $(A)$ with considerable variation in cell size and lobulation of the nucleus. Active erythropoiesis and myelopoiesis showing normal differentiation.

cations when cardiovascular risk factors (especially smoking and obesity) were present. ${ }^{4}$ Therapy is based on risk stratification in which age, a previous vascular event and the number of platelets are taken into consideration. ${ }^{5}$ Young people with ET (age $<60$ years) with no history of thrombosis and a platelet count $<1500 \times 10^{9} / 1$ are considered to be at low risk of thrombosis. This risk is high in patients with a previous history of thrombosis or age $>60$ years. Previous reports show that cytoreductive therapy is not needed in low-risk patients with ET. These reports encourage the use of low-dose aspirin in the presence of vasomotor symptoms or general indications for aspirin use. High-risk patients with ET should be treated with cytoreductive therapy and ASA. ${ }^{6}$ One of the latest studies showed that hydroxyurea plus low-dose aspirin was superior to anagrelide plus low-dose aspirin for patients with essential thrombocythemia at high risk for vascular events. ${ }^{6}$

Ruggeri et al. retrospectively studied the postoperative outcomes in patients with polycythemia vera and essential thrombocythemia. Antiplatelet drugs seemed to be the optimal choice in patients with essential thrombocythemia for antithrombotic prophylaxis in comparison with unfractionated heparin or low-molecular-weight heparin. ${ }^{7}$ However this approach should be weighed against the surgery-specific bleeding risk. Prospective studies are needed to define optimal antithrombotic management in these patients.

We conclude that ET is a risk factor for CHD that should be treated with aspirin. If a patient needs $\mathrm{CABG}$, aspirin has to be continued because of the 
high risk of thrombo-embolic events in high-risk ET patients.

\section{References}

1 Schafer AI. Thrombocytosis. N Engl J Med. 2004;350:1211-9.

2 Hehlmann R, Jahn M, Baumann B, Köpcke W. Essential thrombocythemia: clinical characteristics and course of 61 cases. Cancer. 1988; 61:2487-96.

3 Tefferi A, Fonseca R, Pereira D, Hoagland H. A long-term retrospective study of young women with essential thrombocythemia. Mayo Clin Proc. 2001;76:22.

4 Watson KV, Key N. Vascular complications of essential thrombocythaemia: A link to cardiovascular risk factors. Br J Haematol. 1993;83:198
5 Ruggeri M, Tosetto A, Rodeghiero F, Finazzi G, Barbui T. The management of 'low risk' and 'intermediate risk' patients with primary thrombocythemia. Br J Haematol. 1999;106:834.

6 Harrison CN, Campbell PJ, Buck G, Wheatley K, East CL, Bareford $\mathrm{D}$, et al. Hydroxyurea compared with anagrelide in highrisk essential thrombocythemia. N Engl J Med 2005;353:3345 .

7 Ruggeri M, Rodeghiero F, Tosetto A, Castaman G, Scognamiglio F, Finazzi G, et al.; for the Gruppo Italiano Malattie Ematologiche dell'Adulto (GIMEMA) Chronic Myeloproliferative Diseases Working Party. Postsurgery outcomes in patients with polycythemia vera and essential thrombocythemia: a retrospective survey. Blood. 2008;111:666-71. 